Check for updates

Cite this: Soft Matter, 2019, 15,8512

Received 2nd September 2019, Accepted 13th October 2019

DOI: 10.1039/c9sm01779h

rsc.li/soft-matter-journal

\title{
Preparation of colloidal molecules with temperature-tunable interactions from oppositely charged microgel spheres $\dagger$
}

\author{
Linda K. Månsson, (DD ${ }^{a b}$ Tym de Wild, (D) ${ }^{a}$ Feifei Peng, (D) ${ }^{\text {ab }}$ Stefan H. Holm, (D) bc \\ Jonas $O$. Tegenfeldt (iD ${ }^{\text {bc }}$ and Peter Schurtenberger (iD) *abd
}

\begin{abstract}
The self-assembly of small colloidal clusters, so-called colloidal molecules, into crystalline materials has proven extremely challenging, the outcome often being glassy, amorphous states where positions and orientations are locked. In this paper, a new type of colloidal molecule is therefore prepared, assembled from poly(N-isopropylacrylamide) (PNIPAM)-based microgels that due to their well documented softness and temperature-response allow for greater defect tolerance compared to hard spheres and for convenient in situ tuning of size, volume fraction and inter-particle interactions with temperature. The microgels (B) are assembled by electrostatic adsorption onto oppositely charged, smaller-sized microgels (A), where the relative size of the two determines the valency $(n)$ of the resulting core-satellite $A B_{n}$-type colloidal molecules. Following assembly, a microfluidic deterministic lateral displacement (DLD) device is used to effectively isolate $\mathrm{AB}_{4}$-type colloidal molecules of tetrahedral geometry that possess a repulsiveto-attractive transition on crossing the microgels' volume phase transition temperature (VPTT). These soft, temperature-responsive colloidal molecules constitute highly promising building blocks for the preparation of new materials with emergent properties, and their optical wavelength-size makes them especially interesting for optical applications.
\end{abstract}

\section{Introduction}

It is widely acknowledged that engineered anisotropy with respect to shape and interactions is a powerful approach for realising the self-assembly of colloids into new crystalline materials with new properties, ${ }^{1-8}$ and huge effort has been directed towards the preparation of various anisotropic colloidal building blocks - rods, ${ }^{9-14}$ spindels, ${ }^{15-17}$ mushroom caps and bowls, ${ }^{18-23}$ cubes, $^{24,25}$ dumbbells ${ }^{26-28}$ and peanuts, ${ }^{29,30}$ just to mention a few - and towards understanding the laws that govern their organisation. To expand the assortment of anisotropic colloids, inspired by molecules whose organisation is dictated by the valency and directionality set by the molecular orbitals, quite recently the colloidal analogues were prepared through assembly of spherical colloids into small clusters mimicking space-filling

\footnotetext{
${ }^{a}$ Division of Physical Chemistry, Lund University, POB 124, SE-22100 Lund, Sweden. E-mail: peter.schurtenberger@fkem1.lu.se

${ }^{b}$ NanoLund, POB 118, SE-22100 Lund, Sweden

${ }^{c}$ Division of Solid State Physics, Lund University, POB 118, SE-22100 Lund, Sweden

${ }^{d}$ Lund Institute of Advanced Neutron and X-ray Science (LINXS), Scheelevägen 19, SE-22370 Lund, Sweden

$\dagger$ Electronic supplementary information (ESI) available. See DOI: 10.1039/ c9sm01779h
}

molecule models. These molecule-like clusters are collectively known as 'colloidal molecules'. 31,32

Several methods for the preparation of colloidal molecules have been reported in the literature, including chemical $^{33-36}$ and physical routes ${ }^{21,37-47}$ as well as template strategies. ${ }^{48-50}$ Despite having been around for more than a decade, however, the success in assembling colloidal molecules into crystalline materials has been severely limited, as is evident from the astonishing lack of publications in the area; only very recently was their assembly demonstrated experimentally, when a colloidal $\mathrm{MgCu}_{2}$ alloy was prepared using DNA-mediated assembly from a binary mixture of preassembled tetrahedral colloidal molecules and spheres. ${ }^{51}$ The difficulty in assembling colloidal molecules into periodic structures - as compared to their spherical constituents - lies within the greater probability of kinetic trapping of particle positions and orientations in the assembling structure, resulting in glassy, amorphous states. This puts greater demands on our ability to tune the interparticle interactions during self-assembly, which is extremely difficult to realise with colloidal molecules assembled from traditional silica, polystyrene (PS) or poly(methyl methacrylate) (PMMA) (charged) hard sphere-type building blocks.

In the present paper, the aforementioned problems associated with colloidal molecule self-assembly are addressed. 
This is realised by reaching beyond the classical (charged) hard sphere building blocks, by fabricating colloidal molecules from soft microgel particles ${ }^{52-59}$ that possess a greater defect tolerance in crystallisation compared to the hard counterparts and whose size, volume fraction and interactions can all be conveniently tuned in situ with temperature. Microgels are spherical, waterswollen, crosslinked polymer particles of colloidal dimensions. Due to the identity of the polymers used in the present paper temperature-responsive poly( $N$-isopropylacrylamide) (PNIPAM) and poly( $N$-isopropylmethacrylamide) (PNIPMAM) with a lower critical solution temperature (LCST) of $32^{60,61}$ and $\sim 45{ }^{\circ} \mathrm{C},{ }^{62-67}$ respectively - the corresponding microgels posses a so-called volume phase transition temperature (VPTT).$^{68}$ Below the VPTT, the microgels are highly water-swollen and soft, and their interactions are repulsive in nature due to a combination of sterics conveyed by dangling polymer chains and electrostatics originating from charged moieties in the polymer network. Increasing the temperature across the VPTT, the change of solvent quality leads to water expulsion and particle shrinkage. Above the VPTT, in the collapsed state, van der Waals interactions are important and - in case of sufficient charge screening the microgels experience an attractive force at small separations. Since softness, size, volume fraction and interaction behaviour can all be conveniently manipulated via temperature, microgels have been widely used as versatile model systems for various phase behaviour studies. ${ }^{69-78}$ Besides their well-known VPTT, another important characteristic of PNIPAM and PNIPMAM microgels is their dense core-fuzzy shell morphology, ${ }^{79-82}$ a result of the faster reaction kinetics of the crosslinker - typically $N, N^{\prime}$-methylenebis(acrylamide) (BIS) - compared to the main monomer. ${ }^{83}$

As attractive electrostatic interactions have previously proven to successfully yield small clusters through heteroaggregation, ${ }^{43,45,84-86}$ in the present paper colloidal molecules are prepared from a binary mixture of oppositely charged microgels - one smaller and cationic (labeled A), and one larger and anionic (labeled B) - into small, narrowly size-distributed, colloidal molecule-like $\mathrm{AB}_{n}$-type clusters with a core-satellite structure (Fig. 1), where the valency $n$ can be controlled via the B:A size ratio. With satellites (B) in large excess with respect to cores (A) in order to suppress random aggregation, and at high ionic strength where the electrostatic attraction is screened, assembly is initiated through dialysis that serves to turn on the attraction. Following assembly, microfluidic deterministic lateral displacement (DLD) technology is used as an efficient tool to isolate $\mathrm{AB}_{4}$-type colloidal molecules - promising candidates for assembly of the long-sought photonic diamond lattice, as predicted by simulations ${ }^{87-90}$ - through removal of excess satellites. Finally, the temperature-dependent interaction behaviour of the colloidal molecules is demonstrated using confocal microscopy. Their temperature response with respect to interactions, size and volume fraction, together with their softness and defect tolerance, make these colloidal molecules highly promising building blocks for self-assembly, relieving some of the issues typically associated with assembly of hard sphere-type colloidal molecules.

\section{Preparation of small clusters via electrostatic assembly: some general considerations}

Because of attractive electrostatic interactions, oppositely-charged colloids can, under the right conditions, assemble into small clusters with a core-satellite structure. In such a system, the electrostatic attractions are specific to particles of opposite charge whereas the individual sets of particles are stabilised by electrostatic repulsion. Before entering into our experimental results that build on this method for cluster generation, we briefly discuss the factors that influence the cluster size, the cluster size distribution and the cluster geometry - this in order to rationalise the design of our experiments. Here, 'cluster size' refers to the valency or coordination number, i.e. the number $n$ of satellites (B) that are electrostatically adsorbed onto a core (A), and we use the $\mathrm{AB}_{n}$ annotation to describe the overall cluster composition. For most applications - crystallisation, for instance - it is beneficial to obtain a high yield of a single type (size) of cluster.

The target colloidal molecules in the present study are $\mathrm{AB}_{4}-$ type clusters of tetrahedral geometry. Colloids with a tetrahedral arrangement of attractive sites, mimicking the $\mathrm{sp}^{3}$-hybridised carbon center in methane, have received much attention as simulations have predicted their ability to assemble into the diamond lattice $e^{87-90}$ that exhibits a complete photonic bandgap in the optical regime. ${ }^{91}$ The ability to prepare the necessary building blocks, and to be able

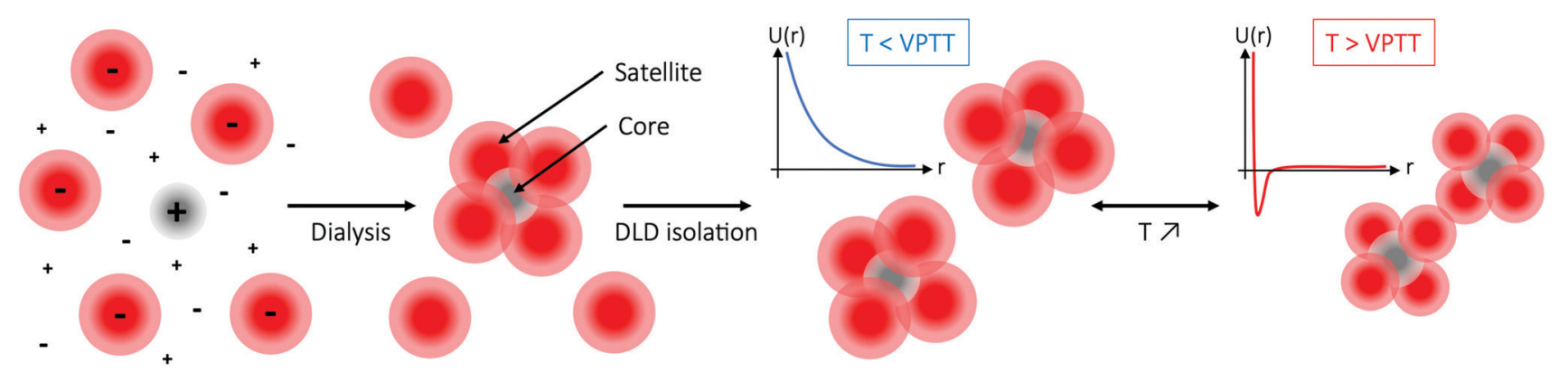

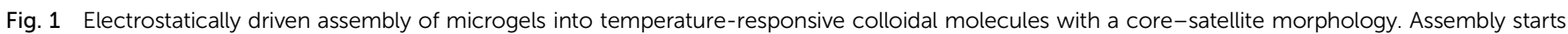

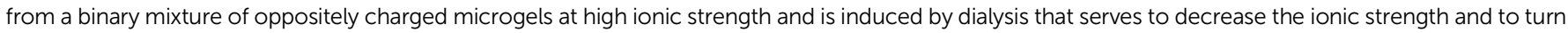

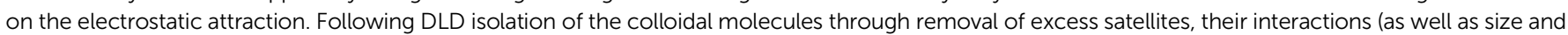
volume fraction) can be tuned via temperature from soft repulsive ( $T<$ VPTT) to hard sphere-like with an added short-range attraction ( $T>$ VPTT). 
to control their interactions and self-assembly, is consequently of large interest and high priority to material scientists. The question then arises, for which $\mathrm{B}$ : A size ratio is $\mathrm{AB}_{4}$ clusters obtained?

It is quite intuitive that the $\mathrm{B}$ : A size ratio is of great importance for the size of the clusters. If considering hard spheres, for simplicity, a simple trigonometric calculation reveals that four large spheres of radius $R$, in a close-packed tetrahedral arrangement (face-centered cubic, FCC), can accommodate a small sphere of radius $r=(\sqrt{3 / 2}-1) R \approx 0.225 R$ inside the tetrahedral void. This means that four large spheres can just fit around the central small sphere when the size ratio $R / r=1 /(\sqrt{3 / 2}-1) \approx 4.45$; for $R / r>4.45$ only three spheres can fit. Decreasing $R / r$ from 4.45 merely causes loss of close-packing up until a certain point at which a fifth large sphere can fit and - assuming that the large spheres can 'roll' on the surface of the smaller one - close-packing is restored for geometrical reasons.

Discussing hard spheres is a useful starting point, but most colloids are never truly hard. For example, the presence of longrange, repulsive electrostatic interactions that (in the case of low ionic strength) reach far beyond the particle surface makes the effective particle size and the preferred satellite center-tocenter distance considerably larger than observed for hard spheres that can be packed until their surfaces touch. This was observed by Demirörs and co-workers who assembled oppositely charged PMMA particles of $R / r=2.19$ in an intermediate-dielectric constant medium and varied the strength of the satellite repulsion via the magnitude of the particle charge: a decrease of $n$ was observed with increasing satellite charge. ${ }^{45}$ When instead the range of the electrostatic interactions was tuned through the addition of salt, a peak in the cluster distribution was observed with increasing salt due to an interplay between satellite-satellite repulsion and satellite-core attraction.

Whereas all of the clusters obtained in Demirörs' study had regular geometries ${ }^{45}$ - explained by the strong satellite-satellite repulsion - the typical situation is that strong, short-range, van der Waals attractions cause the satellites to randomly and irreversibly stick, or 'park', on the cores, thus precluding the formation of symmetric clusters; in this sense, microgels are beneficial since they lack strong short-ranged van der Waals attractions. Despite its stochastic nature, the assembly of sticky particles can yield remarkably narrow size distributions. Schade et al. showed by simulations that for a $R / r=1+\sqrt{2} \approx 2.41$ size ratio, the yield of loosely packed, distorted tetrahedral clusters reaches $100 \% .{ }^{43}$ At this critical size ratio, the upper bound set by packing constraints equals a long-time lower bound that can be derived from solutions to the mathematical spherical covering problem. In the same study, for $R / r=2.45,90 \%$ tetramers were experimentally obtained from assembly of oppositely charged PS spheres. The deviation from the simulated distribution was explained by the presence of vacant 'parking spaces' on the cores even at long times, and by particle size polydispersity. Irregular cluster geometries were also observed by Tagliazucchi and co-workers who used PNIPMAM microgels as satellites and PS particles as cores. ${ }^{84}$
As different from the particles used in the aforementioned studies, it is important to bear in mind that both the cores and the satellites used in the present study are soft in nature and can interpenetrate, compress and deform. ${ }^{92-94}$ The role of softness was recently addressed by Colla et al. who used molecular dynamics (MD) simulations to study the electrostatically driven assembly in a fully symmetric, coarse-gained system of oppositely charged microgels. ${ }^{95}$ Here, the softnessrelated, elastic short-range repulsion was described by the Hertzian potential, ${ }^{76,78,96,97}$ which, most importantly, allows for microgel interpenetration. Through this interpenetration, oppositely charged microgels neutralise each other's charges very efficiently and the minimum of the potential well is (for a moderate to high degree of softness) therefore found well inside the overlap region. This results in the formation of very dense aggregates, thus reducing the system's overall charge inhomogeneity and decreasing the charge ordering commonly seen in charged hard sphere systems.

\section{Materials and methods}

\subsection{Materials}

$N$-Isopropylacrylamide (NIPAM, 97\%, Aldrich), $N$-isopropylmethacrylamide (NIPMAM, 97\%, Aldrich), $N, N^{\prime}$-methylenebis(acrylamide) (BIS, 99\%, Sigma-Aldrich), acrylic acid (AAc, 99.5\%, Acros Organics), allylamine (AL, 98\%, Aldrich), methacryloxyethyl thiocarbamoyl rhodamine B (MRB, Polysciences Inc.), fluorescein O-methacrylate (FMA, 97\%, Aldrich), sodium dodecyl sulfate (SDS, $>99 \%$, Duchefa Biochemie), 2,2'-azobis(2-methylpropionamidine)dihydrochloride (V50, 97\%, Aldrich) and potassium persulfate (KPS, >99\%, Sigma-Aldrich) were all used as received. Water was purified using a Milli-Q water purification system (resistivity 18.2 $\mathrm{M} \Omega \mathrm{cm}$, Millipore).

\subsection{Microgel synthesis}

3.2.1 PNIPAM-co-AAc microgels B474. A mixture of NIPAM (2.87 g, $25.4 \mathrm{mmol}$ ), BIS (0.22 g, $1.4 \mathrm{mmol}, 4.8 \mathrm{~mol} \%$ ), AAc (0.16 g, $2.2 \mathrm{mmol}, 7.6 \mathrm{~mol} \%$ ), MRB (4 mg) and SDS (7 mg) in water $(190.0 \mathrm{ml})$ was stirred and purged with nitrogen for $30 \mathrm{~min}$ at room temperature before increasing the temperature to $70{ }^{\circ} \mathrm{C}$. A solution of KPS (78 $\mathrm{mg}$ in $5.0 \mathrm{ml}$ of water) was then added to start the polymerisation. The reaction was maintained at $70{ }^{\circ} \mathrm{C}$ under nitrogen for $4 \mathrm{~h}$ before cooling down to room temperature. The microgel suspension was filtered through glass wool to remove traces of coagulum, and was thereafter purified by repeated cycles of centrifugation, decantation and redispersion.

3.2.2 PNIPAM-co-AL microgels A115. The synthesis procedure was adapted from literature. ${ }^{98-100}$ A mixture of NIPAM (3.00 g, $26.5 \mathrm{mmol}$ ), BIS (0.21 g, $1.4 \mathrm{mmol}, 4.6 \mathrm{~mol} \%$ ), $\mathrm{AL}$ (0.15 g, $2.6 \mathrm{mmol}, 8.5 \mathrm{~mol} \%$ ) and SDS (42 $\mathrm{mg})$ in water $(180.0 \mathrm{ml})$ was stirred and purged with nitrogen for $1 \mathrm{~h}$ at $60{ }^{\circ} \mathrm{C}$ before raising the temperature to $70{ }^{\circ} \mathrm{C}$. A solution of KPS $(141 \mathrm{mg}$ in $20.0 \mathrm{ml}$ ) was then added to initiate polymerisation, which was allowed to proceed for $4 \mathrm{~h}$ at $70{ }^{\circ} \mathrm{C}$ and under 
nitrogen before cooling down. The suspension was filtered through glass wool and was thereafter purified by dialysis for 1 week.

3.2.3 PNIPMAM-co-AL microgels A220. The same procedure as for the PNIPAM-co-AL microgels A115 was followed but with NIPMAM $(3.00 \mathrm{~g}, 23.6 \mathrm{mmol})$, BIS (0.18 g, $1.2 \mathrm{mmol}$, $4.4 \mathrm{~mol} \%$ ), AL (0.13 g, $2.3 \mathrm{mmol}, 8.5 \mathrm{~mol} \%$ ), SDS (40 mg), water $(180.0 \mathrm{ml})$ and KPS (119 $\mathrm{mg}$ in $20.0 \mathrm{ml})$.

3.2.4 PNIPAM-co-AL microgels A394. A mixture of NIPAM (2.99 g, $26.4 \mathrm{mmol})$, BIS (0.20 g, $1.3 \mathrm{mmol}, 3.1 \mathrm{~mol} \%$ ) and FMA (4.3 mg) in water $(160.0 \mathrm{ml})$ was stirred and purged with nitrogen for $1 \mathrm{~h}$ at $60{ }^{\circ} \mathrm{C}$ before increasing the temperature to $80{ }^{\circ} \mathrm{C}$. A solution of V50 (71 $\mathrm{mg}$ in $10.0 \mathrm{ml})$ was added to start the polymerisation, $3 \mathrm{~min}$ later followed by a mixture of NIPAM $(1.43 \mathrm{~g}, 12.6 \mathrm{mmol})$ and $\mathrm{AL}$ (72 $\mathrm{mg}, 1.3 \mathrm{mmol}, 3.1 \mathrm{~mol} \% \mathrm{AL})$ in water $(10.0 \mathrm{ml})$. The reaction was allowed to proceed for $4 \mathrm{~h}$ at $70{ }^{\circ} \mathrm{C}$ and under nitrogen. The suspension was filtered through glass wool and was then purified by repeated centrifugation rounds.

\subsection{Preparation of colloidal molecules}

Colloidal molecules were prepared by mixing core microgels (A) suspended in $0.1 \mathrm{M} \mathrm{KCl}$ (aq.) with satellite microgels (B) suspended in $0.1 \mathrm{M} \mathrm{KCl}$ (aq.) to yield a final satellite concentration of $\approx 1.1 \mathrm{wt} \%$ and a $\mathrm{B}:$ A number ratio of $\approx(12 \times n): 1$. The mixture was vortexed for a minimum of $4 \mathrm{~h}$ to distribute the cores among the satellites, and was thereafter - in order to induce assembly - dialysed against water, under tumbling, for a minimum of 3 days to ensure complete saturation of the cores by adsorbing satellites.

\subsection{Analysis of colloidal molecule size distributions}

Colloidal molecule size distribution was determined by analytical ultracentrifugation (AUC) using a rotating disk centrifuge (CPS Instruments CPS-24000). At $12144 \mathrm{rpm}, 9$ sucrose solutions of decreasing density from 8 to $2 \mathrm{wt} \%$ (1.6 $\mathrm{ml}$ of each) were layered on top of each other, and after $30 \mathrm{~min}$ the sample to be analysed $(\approx 0.1 \mathrm{ml}$ of $\approx 0.07 \mathrm{wt} \%)$ was injected. The size distribution was obtained by measuring the time required to reach a certain position in the gradient near the outer edge of the disk, where detection takes place. The concentration at this position was measured by light absorbance as a function of time, and was then converted (by the software) to a weight distribution using Mie theory.

\subsection{Isolation of colloidal molecules}

Isolation of colloidal molecules through removal of excess satellites was achieved using a deterministic lateral displacement (DLD) device. A detailed description of the device can be found in the ESI. $\dagger$ A dilute suspension $(\approx 0.06 \mathrm{wt} \%)$ of clusters and excess satellite microgels was introduced through the two sample inlets at the sides of the array, whereas water was introduced through the central inlet. The pressures $(\approx 100 \mathrm{mbar})$ applied to the inlets were fine-tuned to ensure a balanced flow aligned along the direction of the channel. The device was typically run for $4 \mathrm{~h}$, which generated $\approx 40 \mu \mathrm{l}$ of suspension in the cluster outlet. Upon recovery from the outlet reservoir, some of the water was allowed to evaporate in order to increase the cluster concentration.

\subsection{Characterisation techniques}

3.6.1 3D dynamic light scattering (3D DLS). DLS was performed on highly dilute suspensions $\left(10^{-2} \mathrm{wt} \%\right)$ using a 3D light scattering spectrometer (LS instruments AG) that implements a solid state laser light source $(660.0 \mathrm{~nm}, 100 \mathrm{~mW})$. The fluctuations in the intensity of the scattered light were measured in a 3D crosscorrelation configuration ${ }^{101}$ with modulation unit. ${ }^{102} 5 \mathrm{~mm}$ cylindrical NMR-tubes were used as sample cells. 21 correlograms - 3 per angle, each with $60 \mathrm{~s}$ acquisition time - were obtained at scattering angles from 40 to $100^{\circ}$ in steps of $10^{\circ}$. The diffusion coefficient $D$ was obtained by linear regression from $\Gamma=D q^{2}$, with $\Gamma$ the decay rate from a single exponential fit and $q$ the magnitude of the scattering vector. The hydrodynamic radius $\left(R_{\mathrm{H}}\right)$ was calculated from $D$ using the Stokes-Einstein equation, assuming hard spheres of radius $R_{\mathrm{H}}$. Microgel swelling curves were obtained by measuring $R_{\mathrm{H}}$ as a function of temperature in the range 20 to $55{ }^{\circ} \mathrm{C}$. Temperature control of the sample was ensured by regulating the vat temperature using a water circulation thermostat.

3.6.2 Confocal laser scanning microscopy (CLSM). CLSM micrographs were recorded on an inverted confocal laser scanning microscope (Leica DMI6000) with an SP5 tandem scanner operating in resonant mode and using a $100 \times / 1.4 \mathrm{NA}$ oil immersion objective. The microscope is mounted in a thermostated enclosure that enables temperature control with an accuracy of $0.2{ }^{\circ} \mathrm{C}$. The samples under study were kept between two cover glasses separated by a $0.12 \mathrm{~mm}$ spacer (Invitrogen Secure-Seal $^{\mathrm{TM}}$ imaging spacer). Covalent incorporation of fluorescent rhodamine and fluorescein derivatives (MRB and FMA) during microgel synthesis enabled fluorescence CLSM studies. A $543 \mathrm{~nm}$ HeNe and a $488 \mathrm{~nm}$ Ar laser was used to excite the two respective fluorophores.

3.6.3 Electrophoretic mobility measurements. A Malvern Zetasizer Nano-Z (Malvern Instruments Ltd) equipped with a $633 \mathrm{~nm} 4 \mathrm{~mW}$ HeNe laser with automatic laser attenuator was used for electrophoretic mobility $(\mu)$ measurements. Disposable folded capillary cells (Malvern DTS1070) were used as samples cells. Measurements were performed at a fixed scattering angle of $17^{\circ}$ using the M3-PALS laser interferometric technique. The electrophoretic mobility is given as the average of 10 consecutive measurements. Samples were equilibrated for 15 minutes prior to measurements. Sample concentration was $10^{-2} \mathrm{wt} \%$.

\section{Results and discussion}

\subsection{The microgel building blocks}

Soft, temperature-responsive microgels were chosen as building blocks for the preparation of core-satellite, $\mathrm{AB}_{n}$-type colloidal molecules. In a previous publication we already reported on the ability of microgels to serve as temperature-responsive interaction sites - there to mediate the interactions between micron-sized, microgel-decorated oil droplets. ${ }^{103}$ In the present work, anionic, rhodamine-labelled PNIPAM microgels of $R_{\mathrm{H}} 474 \mathrm{~nm}$ (at $20{ }^{\circ} \mathrm{C}$ ) 
Table 1 Overview of selected properties of the four microgel systems used in the present paper

\begin{tabular}{lllllllrr}
\hline Microgel ID & Polymer & $\begin{array}{l}\text { BIS } \\
{[\mathrm{mol} \%]}\end{array}$ & $\begin{array}{l}\text { Co-monomer } \\
{[\mathrm{mol} \%]}\end{array}$ & $\begin{array}{l}\mu \times 10^{-8} @ 20{ }^{\circ} \mathrm{C} \\
{\left[\mathrm{m}^{2} \mathrm{~V}^{-1} \mathrm{~s}^{-1}\right]}\end{array}$ & $\begin{array}{l}R_{\mathrm{H} @ 20}{ }^{\circ} \mathrm{C} \\
{[\mathrm{nm}]}\end{array}$ & $\begin{array}{l}R_{\mathrm{H} @ 55} @{ }^{\circ} \mathrm{C} \\
{[\mathrm{nm}]}\end{array}$ & $\alpha\left(20{ }^{\circ} \mathrm{C}\right)$ & $\mathrm{B} 474: \mathrm{Axxx} @ 20{ }^{\circ} \mathrm{C}$ \\
\hline B474 & PNIPAM-co-AAc & 4.8 & 7.6 & -2.46 & 474 & 233 & 8.4 & - \\
A115 & PNIPAM-co-AL & 4.6 & 8.5 & +2.80 & 115 & 70 & 4.4 \\
A220 & PNIPMAM-co-AL & 4.4 & 8.5 & +3.14 & 220 & 113 & 7.4 & 2.12 \\
A394 & PNIPAM-co-AL & 3.1 & 3.1 & +2.47 & 394 & 177 & 11.0 & 1.20
\end{tabular}

were chosen to serve as satellites (B), and will in the following be referred to as B474. PNIPAM microgels were preferred as satellites over poly( $N$-isopropylmethacrylamide) (PNIPMAM)-based ones due to the lower, more easily accessible VPTT (32 versus $45{ }^{\circ} \mathrm{C}$ ). As cores (A), three oppositely charged (cationic) microgel particles were investigated, and were picked based on their size. The three core microgels are in the following referred to as A115, A220 and A394, respectively, where 115, 220 and 394 refer to their respective $R_{\mathrm{H}}$ in units of $\mathrm{nm}$ (at $20{ }^{\circ} \mathrm{C}$ ). The $\mathrm{A} 220$ cores are based on PNIPMAM whereas the other two are PNIPAM-based; the A394 cores are fluorescein-labelled whereas the other two are nonfluorescent. All four microgels were obtained by free radical precipitation polymerisation, ${ }^{5,104-106}$ and are all moderately crosslinked using BIS (3.1-4.8 mol\%). Negative charges where incorporated into B474 through co-polymerisation with AAc, whereas positively charged $\mathrm{AL}$ was used as co-monomer in the synthesis of A115, A220 and A394. Incorporation of the charged co-monomers was confirmed by measurements of the microgels' electrophoretic mobility $\mu$ in water (Table 1). All four microgels were monodisperse and readily crystallize as evident from Bragg diffraction in concentrated suspensions. CLSM micrographs of the fluorescent B474 and A394 microgels are shown in Fig. 2; the small size and poor contrast of the (non-fluorescent) A115 and A220 microgels did not allow for their direct visualisation by (bright-field) CLSM.

The temperature-responsive behaviour of the microgels was studied, first of all, by dynamic light scattering (DLS), by measuring $R_{\mathrm{H}}$ as a function of temperature in the range from 20 to $55{ }^{\circ} \mathrm{C}$ (Fig. $3 \mathrm{~A}$ and Table 1 ). All four microgels possess a volume phase transition on increasing the temperature, for the B474 microgels however less sharp compared to what is commonly reported for pure PNIPAM. Similar swelling curves for PNIPAM-co-AAc microgels have been reported, and were linked
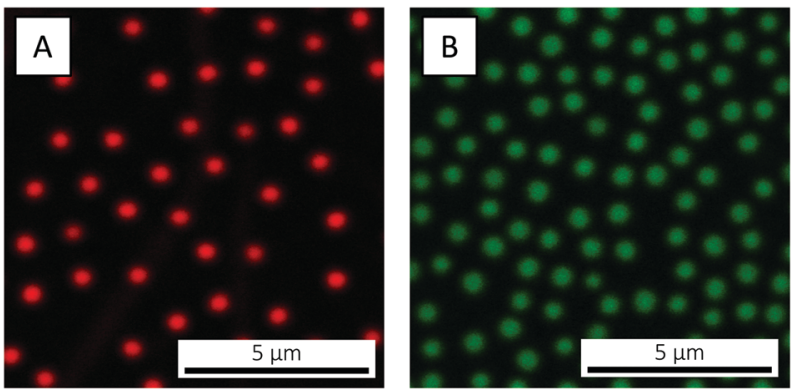

Fig. 2 CLSM micrographs of (A) the B474 satellites and (B) the A394 cores, adsorbed to the cover slip at $20^{\circ} \mathrm{C}$. In both cases the microgels are suspended in water and the concentration is $0.1 \mathrm{wt} \%$. to the presence of charged co-monomer moieties in the particle interior, which counteracts the temperature-induced collapse of the polymer network. ${ }^{109}$ However, recent results indicate that the effect is instead primarily due to the extended conformation of the dangling ends at the microgels' periphery in the strong electric field induced by the presence of the charges. ${ }^{110}$

From the measured $R_{\mathrm{H}}$ values, the swelling ratio $\alpha-\mathrm{a}$ measure of a particle's swelling capacity - can be calculated. $\alpha(T)$ is here defined as

$$
\alpha(T)=\frac{V_{\text {swollen }}}{V_{\text {collapsed }}}=\left[\frac{R_{\mathrm{H}}^{T}}{R_{\mathrm{H}}^{55}}\right]^{3}
$$
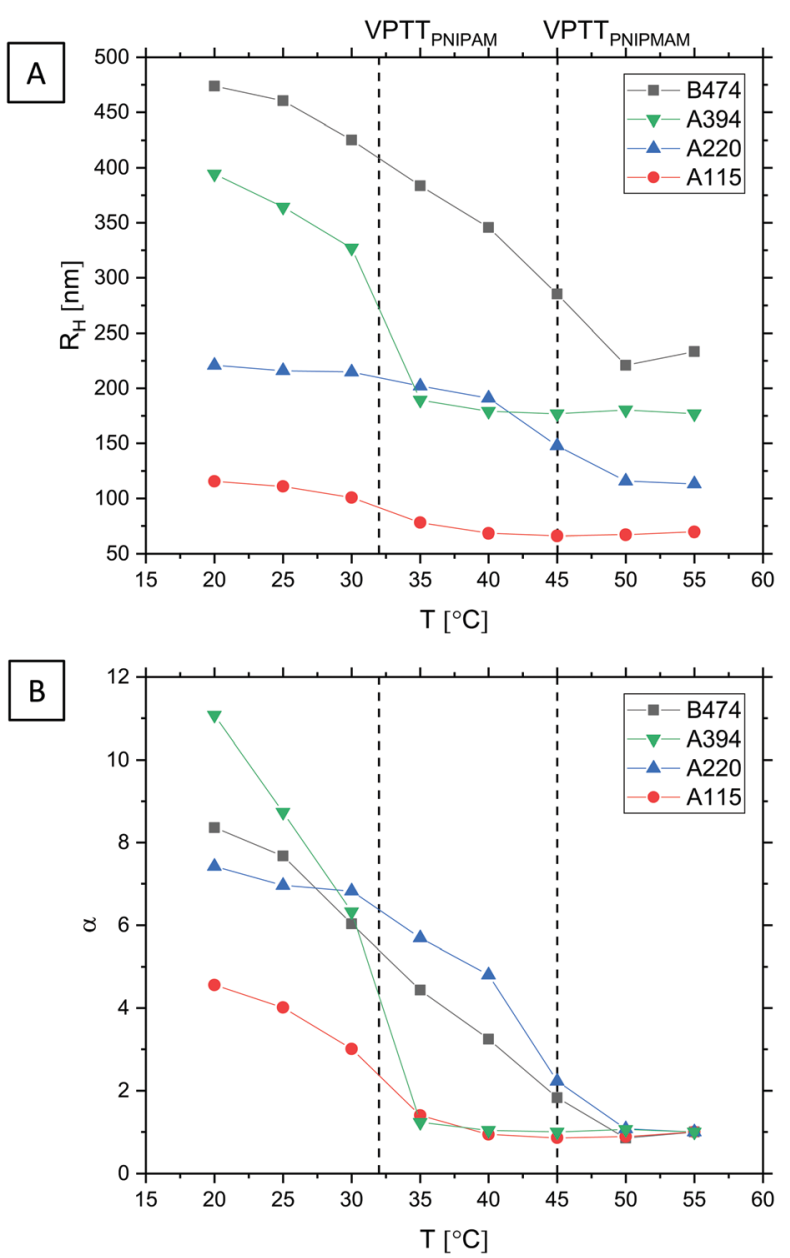

Fig. 3 (A) Microgel swelling curves measured by DLS, showing $R_{\mathrm{H}}$ as a function of $T$. The microgels were dispersed in water. Vertical lines indicate the VPTT of (pure) PNIPAM ${ }^{60,61}$ and PNIPMAM, $64,107,108$ respectively. (B) Evolution of the swelling ratio $\alpha$ with $T$. 


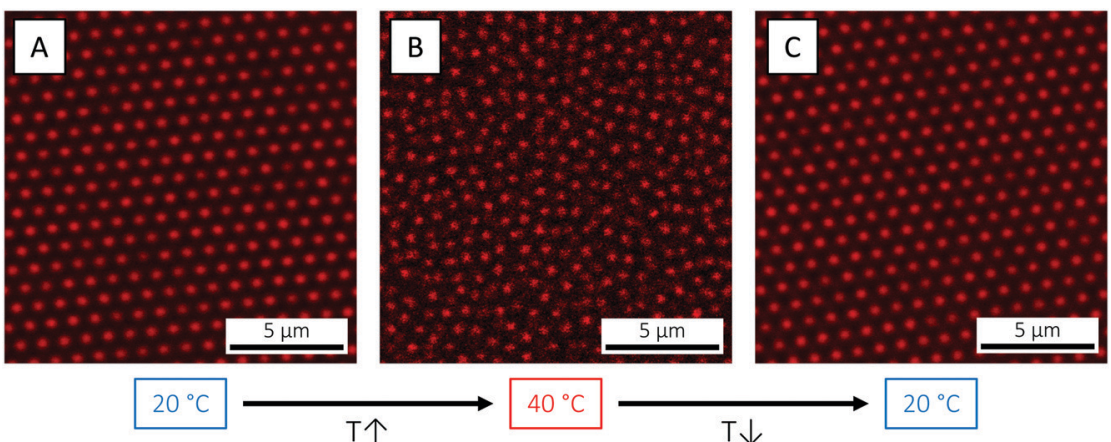

Fig. $4 \mathrm{CLSM}$ micrographs of the B474 microgels at $3.7 \mathrm{wt} \%$ in water, at (A) $20{ }^{\circ} \mathrm{C}\left(T<\right.$ VPTT), (B) $40{ }^{\circ} \mathrm{C}\left(T>\right.$ VPTT) and (C) $20{ }^{\circ} \mathrm{C}$ again, respectively.

where $V_{\text {swollen }}$ and $V_{\text {collapsed }}$ are the particle volume at temperature $T$ and in the fully collapsed state $\left(55^{\circ} \mathrm{C}\right)$, respectively. $\alpha\left(20{ }^{\circ} \mathrm{C}\right)$ varies significantly among the four microgels (Table 1 and Fig. 3B) - from 4.4 for A115 to 11.0 for A394 - which may stem from a combination of factors such as the identity, amount and efficiency of incorporation of monomer, comonomer and crosslinker, as well as various synthesis parameters. The variation in $\alpha\left(20^{\circ} \mathrm{C}\right)$ will not be discussed in more detail, as the aforementioned variables were not systematically investigated in the present paper.

The size (volume) reduction measured by DLS can be directly visualised by confocal laser scanning microscopy (CLSM), here exemplified using the B474 microgels (Fig. 4). At $3.7 \mathrm{wt} \%$ and below the VPTT $\left(20{ }^{\circ} \mathrm{C}\right)$, the highly swollen microgels are in a long-range ordered, crystalline state. As the temperature is raised to $40{ }^{\circ} \mathrm{C}$, however, the particles shrink, their volume fraction reduces and the crystal melts into a fluid state. On returning back to $20{ }^{\circ} \mathrm{C}$, the ordered, crystalline phase is restored. This simple demonstration illustrates how temperature can be effectively used as a tool to explore the phase diagram via in situ tuning of particle size and volume fraction, ${ }^{69}$ which is not possible for hard spheres. As shown elsewhere, ${ }^{111}$ this annealing process can be effectively used to facilitate microgel crystallisation and to remove crystal defects.

In addition to control over size and volume fraction, the use of microgels allows for convenient tuning of the inter-particle interactions through a combination of salt that governs the electrostatic repulsion, and temperature that governs the polymer-solvent interactions and the inter-particle van der Waals attraction. Again, CLSM can be used for direct visualisation of the temperature-response: in Fig. 5, reversible association of the B474 microgels was brought about by increasing the temperature to above the VPTT $\left(40{ }^{\circ} \mathrm{C}\right)$, under acidic conditions $(5 \mathrm{mM} \mathrm{HCl})$ to neutralise the charges of the carboxylic acid groups and to screen the remaining electrostatic repulsion originating from charged initiator residuals. It is worth noticing that $2.5 \mathrm{mM} \mathrm{HCl}$ was not sufficient to suppress the electrostatic stabilisation. Association of the microgels leads to the formation of a volume-spanning network structure, which completely dissociates on returning to temperatures below the VPTT.

\subsection{Preparation of colloidal molecule-like clusters}

Using the oppositely charged satellite B474 and core A115, A220 and A394 microgels, we took on an electrostatically driven assembly approach to prepare core-satellite, $\mathrm{AB}_{n}$-type, colloidal moleculelike clusters (Fig. 1). For mixing of the two species - cores and satellites - two different methods were initially investigated. Common for the two methods is the use of an excess of satellites compared to cores $(\approx(12 \times n): 1 \mathrm{~B}: \mathrm{A}$ number ratio) in order to ensure decoration of the cores by the satellites (and not the other way around) and to suppress formation of unwanted aggregates of alternating satellites and cores. The fundamental difference between the two mixing methods is the ionic strength at which the

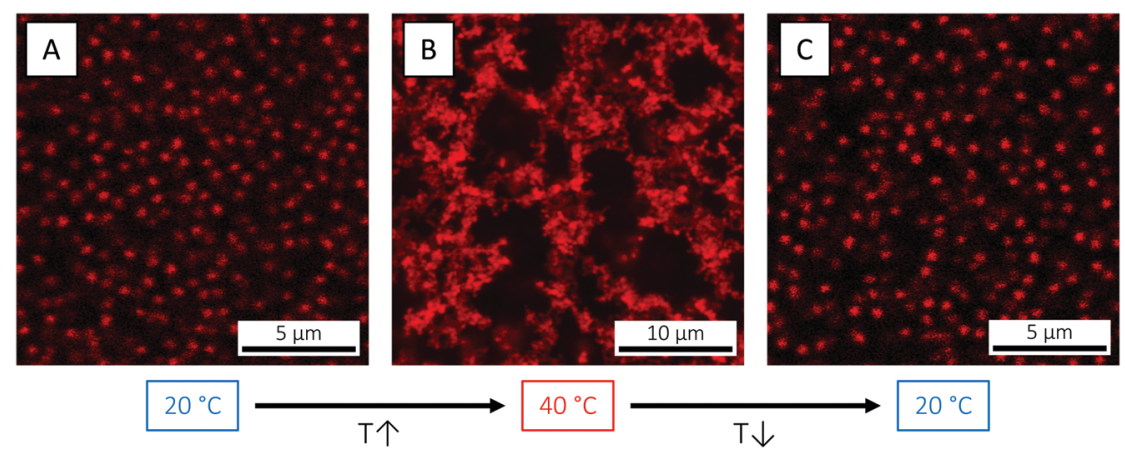

Fig. 5 CLSM micrographs of the B474 microgels at 2.5 wt\% in $5 \mathrm{mM} \mathrm{HCl}$, at (A) 20 ( $T<$ VPTT), (B) 40 ( $T>$ VPTT) and (C) $20^{\circ} \mathrm{C}$ again, respectively. (A) and (C) are snapshots from videos recorded in bulk solution, whereas (B) is a maximum intensity projection constructed from $22 x y$ frames collected over a $z$-distance of $5.29 \mu \mathrm{m}$. 
cores and satellites are mixed. In the first method, the cores were added to the satellites under deionised conditions where electrostatic attractions are present already from the start. In order to suppress aggregate formation, the cores were added only very slowly and at high stirring speed to ensure their rapid distribution among the satellites. In the second method, the cores were instead added to the satellites at high ionic strength $(0.1 \mathrm{M} \mathrm{KCl})$, and not until the resulting suspension had been well mixed and the cores evenly distributed among the satellites, the electrostatic attraction was turned on by dialysis. Whereas the two approaches yielded the same types of small clusters (which we will come back to in the next paragraph), unwanted aggregates (Fig. S1, ESI $\dagger$ ) - sometimes macroscopic - were common in the case of mixing under deionised conditions but were very rare if mixed at high ionic strength. This illustrates the importance of a low local concentration of cores, in combination with a large excess of satellites, in order to suppress aggregate formation. Most likely, as the cores enter the satellite suspension during deionised conditions, they are not distributed among the satellites fast enough, despite slow addition and high stirring speed. In contrast, mixing at high ionic strength allows for the cores to be evenly distributed among the satellites prior to assembly. In that case, suppression of aggregate formation becomes only a matter of having a large enough excess of satellites. The more successful method - mixing at high ionic strength followed by dialysis - was used in all subsequent experiments.

\subsection{Controlling the cluster valency}

Having established a method for core-satellite mixing, the ability to control the valency $n$ of the resulting $\mathrm{AB}_{n}$ clusters via the $\mathrm{B}: \mathrm{A}$ size ratio was investigated. Here, the $\mathrm{B}$ : $\mathrm{A}$ size ratio was varied through the core (A) size, using the A115, A220 and A394 microgels in combination with the B474 satellites. The corresponding B: A size ratios are given in Table 1 . As previously mentioned, $n$ is (for hard particles) governed by geometrical constraints and by the strength and range of the satellite-satellite repulsion. ${ }^{43,45}$ However, as the soft nature of microgels allows for deformation, compression and interpenetration, ${ }^{92-94}$ we acknowledge also that the particle softness and the extent of microgel-microgel overlap might influence $n$. Whereas the effect of microgel softness is outside the scope of the present paper, it is important to keep in mind that for the same value of B:A, the use of soft and hard particles does not necessarily yield clusters of the same $n$. Nevertheless, the B:A size ratio yielding a certain $n$ with hard particles may still serve as a guide. Thus, based on previous work on PS particles, ${ }^{43}$ the A220 cores were selected in order to yield (tetrahedral) $\mathrm{AB}_{4}$-type clusters in combination with the $\mathrm{B} 474$ satellites.

$\mathrm{AB}_{n}$ clusters obtained with B474 in combination with A115, A220 and A394 were qualitatively assessed using CLSM. With the $\mathrm{A} 115$ cores, $\mathrm{AB}_{2}$-type clusters were obtained (Fig. 6A and Video $\mathrm{S} 1$, ESI $\dagger$ ) alongside a very small number of trigonal planar, $\mathrm{AB}_{3}$ ones. All of the $\mathrm{AB}_{2}$ clusters displayed a carbon dioxide-like, linear geometry, likely due to the electrostatic repulsion between the like-charged B474 satellites as also explained elsewhere ${ }^{45}$ this is analogous to the valence shell electron pair repulsion (VSEPR) model proposed for small molecules. The presence of a small number of $\mathrm{AB}_{3}$ clusters is likely reflecting the soft repulsive potential between the satellites, since there remains a finite probability for such $\mathrm{AB}_{3}$ clusters if the strength of the satellitesatellite repulsion is not too large compared to the thermal energy at the point where the ongoing reduction of the ionic strength and thus the increasing core-satellite attraction permanently fixes a given cluster configuration.

On increasing the core size, clusters of higher $n$ were obtained. As aimed for, methane-like, $\mathrm{AB}_{4}$-type clusters of tetrahedral geometry (Fig. 6B and Video S1, ESI $\dagger$ ) were by far the most prevalent ones when the $A 220$ cores were used. $\mathrm{AB}_{3}$ clusters were observed only very rarely, whereas $\mathrm{AB}_{2}$ clusters were absent. As explained elsewhere, ${ }^{43}$ the presence of $\mathrm{AB}_{3}$ clusters can be attributed to unoccupied 'parking spaces' on the cores even at long times.

A further increase in core size, realised using the A394 cores, resulted in sulfur hexafluoride-like, octahedral $\mathrm{AB}_{6}$-type clusters (Fig. 6C and Video S1, ESI $\dagger$ ). Some phosphorus pentafluoridelike, trigonal bipyramidal $\mathrm{AB}_{5}$-type ones were also observed. The electrostatically adsorbed B474 satellites are well separated on the central green cores, giving the assemblies a patchy particlelike appearance.

For all of the A-B combinations investigated in the present paper, regular clusters were obtained: linear dimers, tetrahedral tetramers and octahedral hexamers, respectively. As also argued elsewhere, ${ }^{45}$ we attribute the symmetric distribution of satellites to the electrostatic satellite-satellite repulsion that serves to maximise the satellite-satellite distances. For this maximisation to be possible, the satellites need to be able to rearrange with respect to each other. We believe that such rearrangements are enabled by the slow decrease of ionic strength during the dialysis process that only slowly serves to increase of strength of the core-satellite and satellite-satellite interactions. This initially allows the particles to find their optimal positions, which are slowly 'frozen' as the strength of the interactions increases.

To summarise, the above demonstration that the valency $n$ can be conveniently tuned via the $\mathrm{B}$ : A size ratio makes the electrostatic assembly method for $\mathrm{AB}_{n}$ cluster production highly versatile, as it can be used to generate assemblies ranging from the lowvalency clusters described above to high-valency, raspberry-like ones. Interestingly, despite the soft nature of the microgels and their ability to interpenetrate, compress and deform, ${ }^{92-94}$ the relationship between the $\mathrm{B}$ : A size ratio and $n$ observed for hard particles does not seem to be significantly altered. For the reminder of the present paper, the target tetrahedral, $\mathrm{AB}_{4}$-type clusters that resulted from the assembly of B474 satellites and A220 cores will be the focus.

\subsection{Cluster size distribution analysis}

As some - albeit very rare - $\mathrm{AB}_{3}$-type clusters were observed alongside the $\mathrm{AB}_{4}$ ones following assembly of the $\mathrm{B} 474$ satellites and the A220 cores, the cluster size distribution was quantitatively determined by analytical ultracentrifugation (AUC) in a 8-2 wt\% sucrose gradient using a rotating disc centrifuge that separates clusters of different $n$ based on their different sedimentation velocities $V ; V$ is under Stokes conditions proportional 

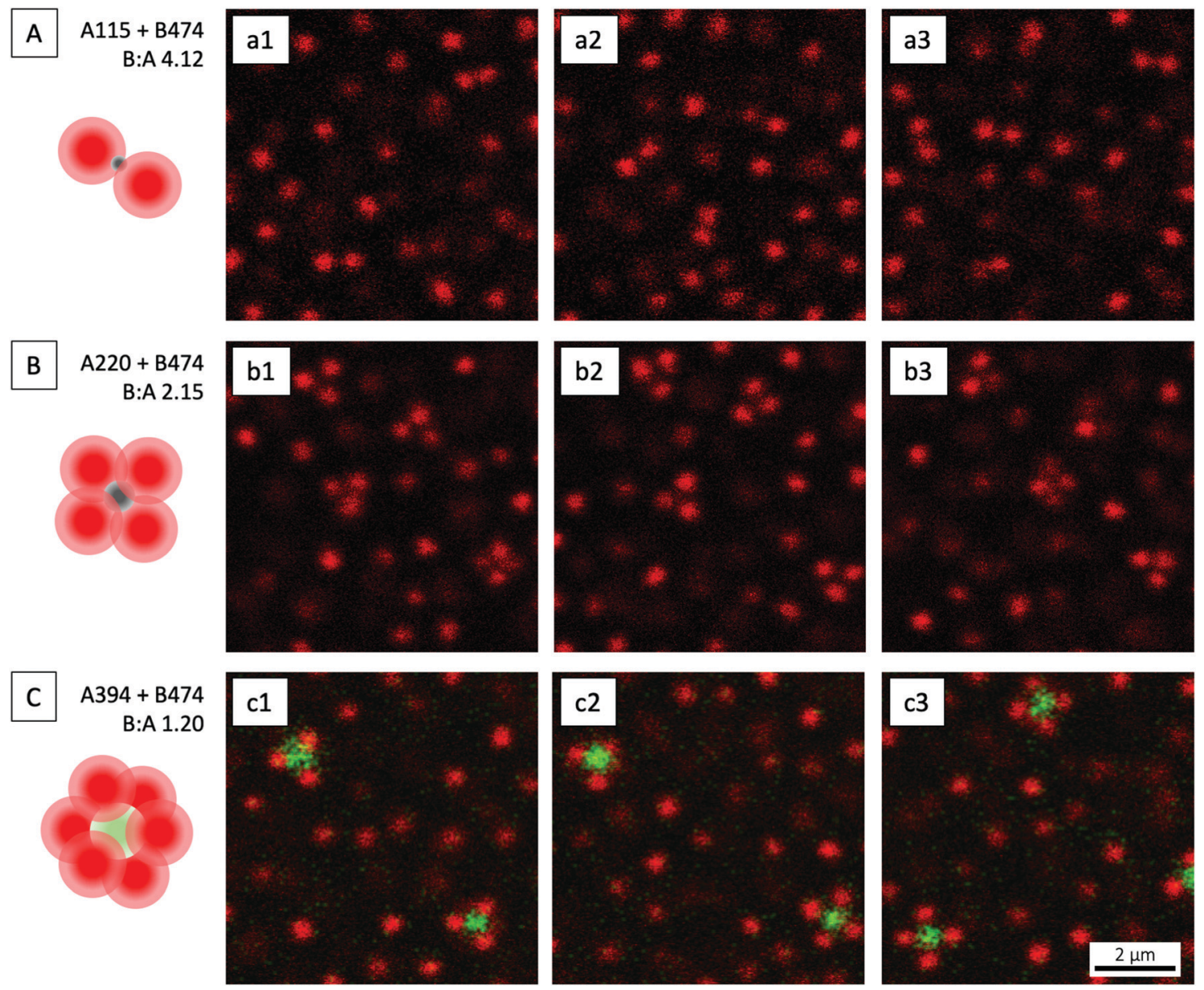

Fig. 6 CLSM micrographs of $\mathrm{AB}_{n}$-type clusters, in an excess of $\mathrm{B} 474$ satellites, at three different $\mathrm{B}$ : $\mathrm{A}$ size ratios: (A) 4.12, (B) 2.15, and (C) 1.20. The micrographs are snapshots from videos recorded in bulk solution. We recommend the reader to watch the corresponding Video S1 (ESI $\dagger$ ) where the geometry of the different clusters is shown more clearly. The schematic drawings show the most prevalent type of cluster in each case.

to the square of the particle diameter $D$ according to $V \propto D^{2}$. A detector located near the outer rim of the rotating disc allows for the different cluster populations to be resolved as individual peaks in a diagram of absorbance versus time, and conversion from turbidity to weight distribution is then done using Mie light scattering theory (based on the assumption of spherical shape). Integration to obtain the peak areas finally gives the relative amount of the different cluster species. Analytical ultracentrifugation has previously been used to assess the distribution of small clusters composed of PS spheres. ${ }^{112,113}$

With AUC, a suspension of pure B474 satellites was first analysed in order to confirm that there were no random aggregates prior to assembly, and indeed only a single peak was observed (Fig. S2, ESI $\dagger$ ). In addition to the satellite peak, a narrow series of peaks corresponding to colloidal molecule-like $\mathrm{AB}_{n}$-type clusters was observed when the suspension harvested from the dialysis bag was analysed (Fig. 7). We conclude that the largest among the peaks corresponds to the many $\mathrm{AB}_{4}$ clusters that were observed by CLSM, whereas the few $\mathrm{AB}_{3}$ clusters give rise to the small peak to its left. A slightly noisy base-line is observed to the right of the $\mathrm{AB}_{4}$ peak, which is probably due to the presence of extremely rare, small aggregates. From integration of the $\mathrm{AB}_{3}$ and
$\mathrm{AB}_{4}$ peaks (Fig. 7), is was determined that the target $\mathrm{AB}_{4}$ clusters constitute $90 \%$ of the total $\left(\mathrm{AB}_{3}-\mathrm{AB}_{4}\right)$ cluster population by weight. As shown for hard particles, ${ }^{43}$ the yield of $\mathrm{AB}_{4}$ microgelbased clusters can likely be boosted further by fine-tuning of the B: A size ratio (and/or of additional parameters that affect $n$ ).

\subsection{Stability of colloidal molecules}

Using microgels, which contain up to $90 \%$ water in the swollen state and therefore possess negligible attractive van der Waals forces, it was presumed that a core-satellite crosslinking strategy needed to be implemented in order to form stable $\mathrm{AB}_{4}$-type, colloidal molecule-like clusters that do not dissociate under the acidic conditions required to induce attractive inter-particle interactions above the VPTT. However, even after 24 hours in $5 \mathrm{mM} \mathrm{HCl} \mathrm{(i.e.} \mathrm{conditions} \mathrm{under} \mathrm{which} \mathrm{the} \mathrm{satellites} \mathrm{are} \mathrm{attrac-}$ tive at $T>$ VPTT), the $\mathrm{AB}_{4}$-type clusters seemed to be, based on CLSM studies, just as prevalent as in pure water (Fig. S3, ESI†). The conclusion is that loss of negative charges due to protonation of the carboxylic acid groups is not sufficient to cause dissociation, possibly as the negative charges originating from persulfate initiator residuals still contribute to sufficient electrostatic attraction between cores and satellites. Not only do the 


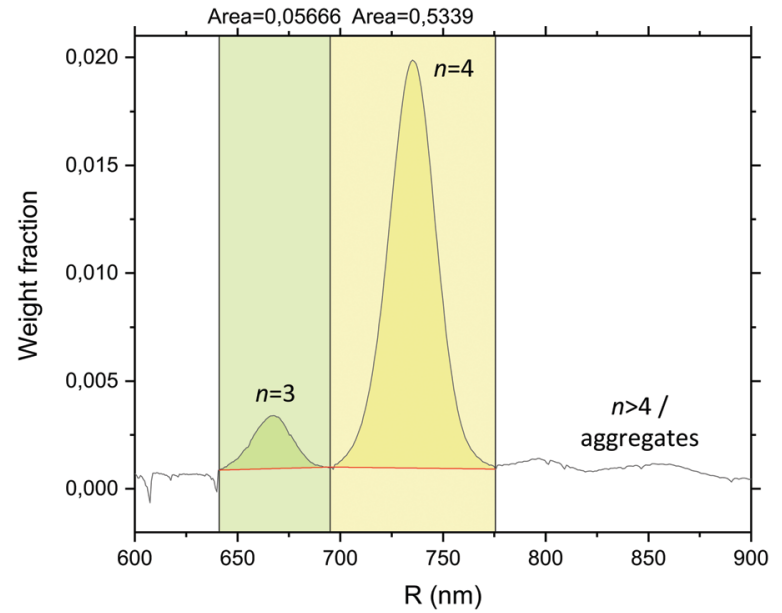

Fig. 7 Particle (cluster) size distribution measured by AUC for the suspension harvested from the dialysis bag following assembly of $B 474$ satellites and $A 220$ cores. The relative abundance (by weight) of $A B_{3}$ and $A B_{4}$ clusters was obtained by integration of the peaks corresponding to the two populations. The percentage of the target $\mathrm{AB}_{4}$ clusters was given by $0.5339 /(0.5339+0.05666)=90 \%$

clusters remain intact in acidic solution, but they also do not dissociate in the presence of salt; after 2 weeks in $0.1 \mathrm{M} \mathrm{KCl}$, the clusters are still as numerous as before, as qualitatively assessed by CLSM (Fig. S3, ESI $\dagger$ ).

In addition to the still prevailing electrostatic attraction under acidic conditions, we believe that the lack of dissociation of the electrostatically assembled clusters under acidic or saline conditions is due to two contributing factors which both stem from the slow dynamics present in polymer systems. First, as also argued elsewhere, ${ }^{85}$ as interpenetration and entanglement of the fuzzy shells of the core and satellite microgels is likely extensive, their mobility with respect to each other is restricted and the core-satellite connection is strengthened; disassembly of the two then requires full disentanglement of these extensively interpenetrating fuzzy shells. Second, for a satellite to desorb from the core, all core-satellite ion pairs need to dissociate at the same time. If only a fraction of the pairs dissociate at a time, there is a high probability that re-pairing occurs before the entire satellite can desorb. In this way, the satellites remains bound to the core by its own inertia. This is much analogous to adsorption of a polyelectrolyte to an oppositely charged surface, which can be regarded as essentially irreversible (over a finite time scale). ${ }^{114}$

Since neither acid nor salt caused dissociation of the clusters, we considered crosslinking not necessary. One can imagine, however, to make use of a 1-ethyl-3-(3-dimethylaminopropyl)carbodiimide (EDC) coupling scheme that serves to connect the carboxylic acid groups of the satellites with the primary amine groups of the cores. ${ }^{115}$

\subsection{Isolation of colloidal molecules}

Due to the large excess of B474 satellites compared to A220 cores ( $\approx 48: 1 \mathrm{~B}:$ A number ratio), assembly of the two naturally yields a mixture of clusters and excess B474 satellites. Isolation of the $\mathrm{AB}_{4}$ clusters through removal of excess satellites was accomplished using DLD technology, ${ }^{116,117}$ a continuous microfluidic particle sorting method that relies on interactions of particles suspended in a fluid with an obstacle array, which is tilted with respect to the flow direction, under laminar flow conditions. While this method in recent years has been shown capable of sorting based on particle properties such as shape, ${ }^{118-120}$ softness, ${ }^{121}$ and polarisability, ${ }^{122}$ its common mode of operation is size-based particle sorting where the method is recognised for its excellent resolution. The size-based sorting relies on a threshold size, the critical diameter $D_{\mathrm{c}}$, which is mainly determined by the array parameters: the post-post distance and the tilt angle between the post array and the flow direction. From the point of entry into the device, particles smaller in size than $D_{\mathrm{c}}$ follow a zigzagged but ultimately straight course as they do not interact sufficiently with the perpendicular posts to be shifted from the overall flow direction. Particles larger than $D_{c}$, on the other hand, are laterally displaced across the streamlines when negotiating each post. In this way, particles smaller and larger than $D_{\mathrm{c}}$ are separated along the length of the device and are finally collected in different outlet reservoirs upon exiting the device. There is a plethora of samples successfully sorted by DLD, including nanoscale colloids and vesicles, ${ }^{123-125}$ blood (platelets, ${ }^{126}$ WBCs, ${ }^{127}$ RBCs, ${ }^{121}$ CTCs, ${ }^{128}$ parasites, ${ }^{129,130}$ ) and other types of samples such as droplets, ${ }^{131}$ bacteria chains ${ }^{132}$ and epithelial cells. ${ }^{133}$

The nominal $D_{\mathrm{c}}$ of the DLD device used in the present work was $D_{\mathrm{c}}=1.18 \mu \mathrm{m}$, as estimated by the empirical expression in ref. 134 . This $D_{\text {c }}$ was large enough to suppress lateral displacement of the excess B474 satellites while still allowing for displacement of the $\mathrm{AB}_{4}$ clusters, consequently achieving successful separation by directing the two species to different outlet reservoirs (Video S2 shows $\mathrm{AB}_{4}$ clusters in outlet reservoir, ESI $\dagger$ ). CLSM micrographs of isolated target $\mathrm{AB}_{n}$-type colloidal molecules are shown in Fig. 8. A detailed description of the DLD device used in this work can be found in the ESI. $\dagger$
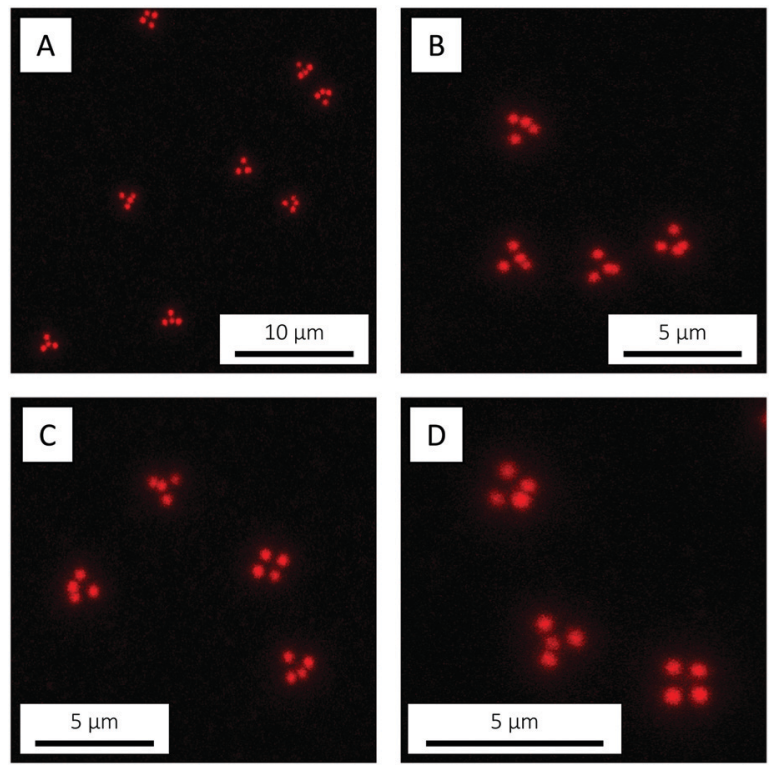

Fig. $8 x y z$ maximum intensity projections, constructed from $40 x y$ frames collected over a $z$-distance of $3.27 \mu \mathrm{m}$ and recorded at $20^{\circ} \mathrm{C}$, showing isolated $\mathrm{AB}_{4}$-type clusters of tetrahedral geometry adsorbed to the glass cover slip. 

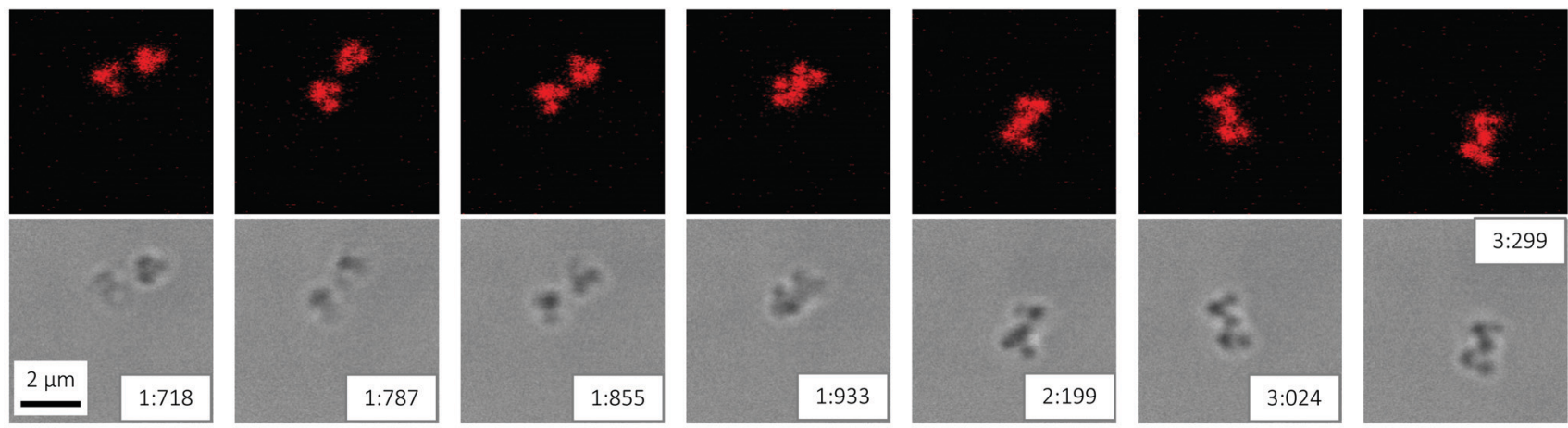

Fig. $9 \mathrm{CLSM}$ micrographs showing the association of two $\mathrm{AB}_{4}$-type clusters, induced by an increase of temperature from $30^{\circ} \mathrm{C}(T<\mathrm{VPTT})$ to $35^{\circ} \mathrm{C}(T>\mathrm{VPTT})$ in $5 \mathrm{mM} \mathrm{HCl}$. The micrographs are snapshots from a video recorded in bulk solution (Video S3, ESI†). The top and bottom panels show fluorescence and brightfield micrographs, respectively.

It is worth mentioning that we also tried to remove the excess free satellites from the clusters using density gradient centrifugation. This however proved difficult due to problems with detection of the bands, as the highly water-swollen microgels scatter light only very weakly. Increasing the concentration was not possible, as it resulted in streaming.

\subsection{Temperature-dependent interactions between colloidal molecules}

The ability to control the inter-particle interactions between the isolated $\mathrm{AB}_{4}$-type, colloidal molecule-like clusters was demonstrated by CLSM, by raising the temperature across the VPTT (from 30 to $35^{\circ} \mathrm{C}$ ) under acidic conditions $(5 \mathrm{mM} \mathrm{HCl})$ in order to sufficiently reduce the electrostatic cluster-cluster repulsion. Before the study, as the sample obtained from DLD was very dilute, the concentration of clusters was increased by evaporation to approximately one third of the volume. Analogous to the observations made for the pure B474 satellite microgels (Fig. 5), increasing the temperature across the VPTT serves to turn on attractive inter-particle interactions that cause association of the clusters. The association of two $\mathrm{AB}_{4}$ clusters is shown in Fig. 9 (Video S3, ESI $\dagger$ ). As for the pure B474 satellites, the association process is completely reversible, and the sample returns to its original state on cooling down. In summary, these observations show that the 'intermolecular' interactions between the $\mathrm{AB}_{4}$ clusters can be tuned in a simple manner through an interplay between electrostatic repulsion, tuned by acid (or salt) concentration, and attractive van der Waals interactions controlled by temperature as an external stimulus.

\section{Conclusions}

Whereas colloidal molecules constitute highly promising building blocks for the preparation of new, ordered structures with novel or improved properties, their typical hard sphere nature has hampered the realisation of large crystals with the necessary degree of perfection, largely due to difficulties with controlling and tuning the inter-particle interactions. The current paper has aimed to address this issue, by replacing the usual hard sphere building blocks with temperature-responsive ones that allows for control of the inter-particle interactions via temperature.

We have here reported on a novel, facile, cheap and easily upscalable bulk method for the preparation of large quantities of temperature-responsive, microgel-based colloidal molecules. We start from a binary mixture of oppositely charged microgels and allow electrostatics to drive their assembly into well-defined, core-satellite $\mathrm{AB}_{n}$-type, colloidal molecule-like clusters, where clusters of a certain $n$ can be obtained through careful selection of cores and satellites of the appropriate relative size. As hypothesised, the inherent response of the microgels to changes in temperature allows for the inter-particle interactions between the clusters to be conveniently controlled with temperature, from soft repulsive below the VPTT to short-range attractive above. We expect that this in situ control of the interactions and of the volume fraction - will greatly facilitate the assembly of colloidal molecules into (dense) crystalline phases. Future work will however have to address the quantitative investigation of the directional inter-particle interactions, the phase behaviour and the self-assembly of the clusters as a function of concentration and temperature. Such studies will naturally require a large amount of the target clusters, which can be realised by simple up-scaling of the fabrication process, by improving the yield of the target cluster type through fine-tuning of the B: A size ratio, ${ }^{43}$ and/or by increasing the throughput of DLD sorting through parallelisation.

For the preparation of open structures, such as the longsought diamond lattice, we now have building blocks of the requisite shape and symmetry at hand. Replacement of our lowoptical density microgels with core-shell microgels comprising an inorganic, metallic or magnetic core ${ }^{135-139}$ provides us with the possibility to prepare full photonic band gap materials in the future.

\section{Conflicts of interest}

There are no conflicts to declare. 


\section{Acknowledgements}

This work was financially supported by the European Research Council (ERC-339678-COMPASS) and by NanoLund at Lund University (p13-2017). The authors thank Emil Gustafsson for assisting in synthesis of the A115 and A220 microgels, Robin Kämpe for writing the MATLAB scripts for DLS analysis, Tommy Cedervall for providing the analytical centrifuge and Jérôme J. Crassous for helpful discussions. DLD device fabrication was carried out in Lund Nano Lab.

\section{References}

1 A. van Blaaderen, Nature, 2006, 439, 545-546.

2 S. C. Glotzer and M. J. Solomon, Nat. Mater., 2007, 6, 557-562.

3 M. J. Solomon, Curr. Opin. Colloid Interface Sci., 2011, 16, 158-167.

4 S. Sacanna and D. J. Pine, Curr. Opin. Colloid Interface Sci., 2011, 16, 96-105.

5 L. Cademartiri, K. J. Bishop, P. W. Snyder and G. A. Ozin, Philos. Trans. R. Soc., A, 2012, 370, 2824-2847.

6 S. Sacanna, D. J. Pine and G.-R. Yi, Soft Matter, 2013, 9, 8096-8106.

7 G. van Anders, N. K. Ahmed, R. Smith, M. Engel and S. C. Glotzer, ACS Nano, 2013, 8, 931-940.

8 A. V. Petukhov, R. Tuinier and G. J. Vroege, Curr. Opin. Colloid Interface Sci., 2017, 30, 54-61.

9 H. Maeda and Y. Maeda, Phys. Rev. Lett., 2003, 90, 018303. 10 A. Mohraz and M. J. Solomon, Langmuir, 2005, 21, 5298-5306.

11 M. J. Solomon and P. T. Spicer, Soft Matter, 2010, 6, 1391-1400.

12 D. Mukhija and M. J. Solomon, Soft Matter, 2011, 7, 540-545.

13 A. Kuijk, A. van Blaaderen and A. Imhof, J. Am. Chem. Soc., 2011, 133, 2346-2349.

14 A. Kuijk, D. V. Byelov, A. V. Petukhov, A. van Blaaderen and A. Imhof, Faraday Discuss., 2012, 159, 181-199.

15 M. Ozaki, S. Kratohvil and E. Matijević, J. Colloid Interface Sci., 1984, 102, 146-151.

16 M. Ozaki and E. Matijević, J. Colloid Interface Sci., 1985, 107, 199-203.

17 T. Ishikawa and E. Matijevic, Langmuir, 1988, 4, 26-31.

18 C. I. Zoldesi and A. Imhof, Adv. Mater., 2005, 17, 924-928.

19 S. H. Im, U. Jeong and Y. Xia, Nat. Mater., 2005, 4, 671-675.

20 E. K. Riley and C. M. Liddell, Langmuir, 2010, 26, 11648-11656.

21 S. Sacanna, W. Irvine, P. M. Chaikin and D. J. Pine, Nature, 2010, 464, 575-578.

22 M. Marechal, R. J. Kortschot, A. F. Demirörs, A. Imhof and M. Dijkstra, Nano Lett., 2010, 10, 1907-1911.

23 W. Jing, S. Du and Z. Zhang, Polymers, 2018, 10, 458.

24 L. Rossi, S. Sacanna, W. T. Irvine, P. M. Chaikin, D. J. Pine and A. P. Philipse, Soft Matter, 2011, 7, 4139-4142.

25 J.-M. Meijer, F. Hagemans, L. Rossi, D. V. Byelov, S. I. Castillo, A. Snigirev, I. Snigireva, A. P. Philipse and A. V. Petukhov, Langmuir, 2012, 28, 7631-7638.

26 P. M. Johnson, C. M. van Kats and A. van Blaaderen, Langmuir, 2005, 21, 11510-11517.
27 M. Marechal and M. Dijkstra, Soft Matter, 2011, 7, 1397-1408.

28 J. D. Forster, J.-G. Park, M. Mittal, H. Noh, C. F. Schreck, C. S. O'Hern, H. Cao, E. M. Furst and E. R. Dufresne, ACS Nano, 2011, 5, 6695-6700.

29 T. Sugimoto, M. M. Khan, A. Muramatsu and H. Itoh, Colloids Surf., A, 1993, 79, 233-247.

30 S. H. Lee, S. J. Gerbode, B. S. John, A. K. Wolfgang, F. A. Escobedo, I. Cohen and C. M. Liddell, J. Mater. Chem., 2008, 18, 4912-4916.

31 A. van Blaaderen, Science, 2003, 301, 470-471.

32 E. Duguet, A. Désert, A. Perro and S. Ravaine, Chem. Soc. Rev., 2011, 40, 941-960.

33 W. K. Kegel, D. Breed, M. Elsesser and D. J. Pine, Langmuir, 2006, 22, 7135-7136.

34 J.-W. Kim, R. J. Larsen and D. A. Weitz, Adv. Mater., 2007, 19, 2005-2009.

35 A. Perro, E. Duguet, O. Lambert, J.-C. Taveau, E. BourgeatLami and S. Ravaine, Angew. Chem., Int. Ed., 2009, 48, 361-365.

36 J.-G. Park, J. D. Forster and E. R. Dufresne, Langmuir, 2009, 25, 8903-8906.

37 L. Hong, A. Cacciuto, E. Luijten and S. Granick, Nano Lett., 2006, 6, 2510-2514.

38 D. J. Kraft, W. S. Vlug, C. M. van Kats, A. van Blaaderen, A. Imhof and W. K. Kegel, J. Am. Chem. Soc., 2008, 131, 1182-1186.

39 D. J. Kraft, J. Groenewold and W. K. Kegel, Soft Matter, 2009, 5, 3823-3826.

40 S. Sacanna, L. Rossi and D. J. Pine, J. Am. Chem. Soc., 2012, 134, 6112-6115.

41 D. J. Kraft, R. Ni, F. Smallenburg, M. Hermes, K. Yoon, D. A. Weitz, A. van Blaaderen, J. Groenewold, M. Dijkstra and W. K. Kegel, Proc. Natl. Acad. Sci. U. S. A., 2012, 109, 10787-10792.

42 Y. Wang, Y. Wang, D. R. Breed, V. N. Manoharan, L. Feng, A. D. Hollingsworth, M. Weck and D. J. Pine, Nature, 2012, 491, 51-55.

43 N. B. Schade, M. C. Holmes-Cerfon, E. R. Chen, D. Aronzon, J. W. Collins, J. A. Fan, F. Capasso and V. N. Manoharan, Phys. Rev. Lett., 2013, 110, 148303.

44 Y. Wang, Y. Wang, X. Zheng, G.-R. Yi, S. Sacanna, D. J. Pine and M. Weck, J. Am. Chem. Soc., 2014, 136, 6866-6869.

45 A. F. Demirörs, J. C. Stiefelhagen, T. Vissers, F. Smallenburg, M. Dijkstra, A. Imhof and A. van Blaaderen, Phys. Rev. X, 2015, 5, 021012.

46 Q. Yuan, J. Gu, Y.-N. Zhao, L. Yao, Y. Guan and Y. Zhang, ACS Macro Lett., 2016, 5, 565-568.

47 L. Yao, Q. Li, Y. Guan, X. Zhu and Y. Zhang, ACS Macro Lett., 2018, 7, 80-84.

48 O. Velev, K. Furusawa and K. Nagayama, Langmuir, 1996, 12, 2374-2384.

49 Y. Yin, Y. Lu, B. Gates and Y. Xia, J. Am. Chem. Soc., 2001, 123, 8718-8729.

50 V. N. Manoharan, M. T. Elsesser and D. J. Pine, Science, 2003, 301, 483-487. 
51 É. Ducrot, M. He, G.-R. Yi and D. J. Pine, Nat. Mater., 2017, 16, 652-657.

52 R. Pelton and P. Chibante, Colloids Surf., 1986, 20, 247-256.

53 B. R. Saunders and B. Vincent, Adv. Colloid Interface Sci., 1999, 80, 1-25.

54 R. Pelton, Adv. Colloid Interface Sci., 2000, 85, 1-33.

55 M. Das, H. Zhang and E. Kumacheva, Annu. Rev. Mater. Res., 2006, 36, 117-142.

56 L. A. Lyon and A. Fernandez-Nieves, Annu. Rev. Phys. Chem., 2012, 63, 25-43.

57 H. Kawaguchi, Polym. Int., 2014, 63, 925-932.

58 J. Brijitta and P. Schurtenberger, Curr. Opin. Colloid Interface Sci., 2019, 40, 87-103.

59 M. Karg, A. Pich, T. Hellweg, T. Hoare, L. A. Lyon, J. J. Crassous, D. Suzuki, R. A. Gumerov, S. Schneider and I. I. Potemkin, et al., Langmuir, 2019, 35, 6231-6255.

60 M. Heskins and J. E. Guillet, J. Macromol. Sci., Chem., 1968, 2, 1441-1455.

61 H. G. Schild, Prog. Polym. Sci., 1992, 17, 163-249.

62 E. I. Tiktopulo, V. N. Uversky, V. B. Lushchik, S. I. Klenin, V. E. Bychkova and O. B. Ptitsyn, Macromolecules, 1995, 28, 7519-7524.

63 N. A. Platé, T. L. Lebedeva and L. I. Valuev, Polym. J., 1999, 31, 21-27.

64 I. Berndt and W. Richtering, Macromolecules, 2003, 36, 8780-8785.

65 M. S. Sánchez, L. Hanyková, M. Ilavský and M. M. Pradas, Polymer, 2004, 45, 4087-4094.

66 Y. Wu, F. Meersman and Y. Ozaki, Macromolecules, 2006, 39, 1182-1188.

67 Y. Tang, Y. Ding and G. Zhang, J. Phys. Chem. B, 2008, 112, 8447-8451.

68 M. Shibayama and T. Tanaka, in Responsive Gels: Volume Transitions I, ed. K. Dušek, Springer, Berlin, Heidelberg, 1993, ch. 1, pp. 1-62.

69 J. Wu, B. Zhou and Z. Hu, Phys. Rev. Lett., 2003, 90, 048304.

70 J. Zhou, G. Wang, L. Zou, L. Tang, M. Marquez and Z. Hu, Biomacromolecules, 2008, 9, 142-148.

71 G. Romeo, A. Fernandez-Nieves, H. M. Wyss, D. Acierno and D. A. Weitz, Adv. Mater., 2010, 22, 3441-3445.

72 A. Zaccone, J. J. Crassous, B. Béri and M. Ballauff, Phys. Rev. Lett., 2011, 107, 168303.

73 P. Holmqvist, P. Mohanty, G. Nägele, P. Schurtenberger and M. Heinen, Phys. Rev. Lett., 2012, 109, 048302.

74 S. Nöjd, P. S. Mohanty, P. Bagheri, A. Yethiraj and P. Schurtenberger, Soft Matter, 2013, 9, 9199-9207.

75 A. Zaccone, J. J. Crassous and M. Ballauff, J. Chem. Phys., 2013, 138, 104908.

76 D. Paloli, P. S. Mohanty, J. J. Crassous, E. Zaccarelli and P. Schurtenberger, Soft Matter, 2013, 9, 3000-3004.

77 P. J. Yunker, K. Chen, M. D. Gratale, M. A. Lohr, T. Still and A. Yodh, Rep. Prog. Phys., 2014, 77, 056601.

78 P. S. Mohanty, D. Paloli, J. J. Crassous, E. Zaccarelli and P. Schurtenberger, J. Chem. Phys., 2014, 140, 094901.

79 I. Varga, T. Gilányi, R. Meszaros, G. Filipcsei and M. Zrinyi, J. Phys. Chem. B, 2001, 105, 9071-9076.
80 B. R. Saunders, Langmuir, 2004, 20, 3925-3932.

81 M. Stieger, W. Richtering, J. S. Pedersen and P. Lindner, J. Chem. Phys., 2004, 120, 6197-6206.

82 M. Reufer, P. Daz-Leyva, I. Lynch and F. Scheffold, Eur. Phys. J. E: Soft Matter Biol. Phys., 2009, 28, 165-171.

$83 \mathrm{X} . \mathrm{Wu}, \mathrm{R}$. Pelton, A. Hamielec, D. Woods and W. McPhee, Colloid Polym. Sci., 1994, 272, 467-477.

84 M. Tagliazucci, F. Zou and E. A. Weiss, J. Phys. Chem. Lett., 2014, 5, 2775-2780.

85 D. Go, T. E. Kodger, J. Sprakel and A. J. C. Kuehne, Soft Matter, 2014, 10, 8060-8065.

86 A. M. Mihut, B. Stenqvist, M. Lund, P. Schurtenberger and J. J. Crassous, Sci. Adv., 2017, 3, e1700321.

87 Z. Zhang, A. S. Keys, T. Chen and S. C. Glotzer, Langmuir, 2005, 21, 11547-11551.

88 E. G. Noya, C. Vega, J. P. Doye and A. A. Louis, J. Chem. Phys., 2010, 132, 234511.

89 F. Romano, E. Sanz and F. Sciortino, J. Chem. Phys., 2011, 134, 174502.

90 D. Morphew, J. Shaw, C. Avins and D. Chakrabarti, ACS Nano, 2018, 12, 2355-2364.

91 K. Ho, C. T. Chan and C. M. Soukoulis, Phys. Rev. Lett., 1990, 65, 3152-3155.

92 N. Boon and P. Schurtenberger, Phys. Chem. Chem. Phys., 2017, 19, 23740-23746.

93 P. S. Mohanty, S. Nöjd, K. van Gruijthuijsen, J. J. Crassous, M. Obiols-Rabasa, R. Schweins, A. Stradner and P. Schurtenberger, Sci. Rep., 2017, 7, 1487.

94 G. M. Conley, P. Aebischer, S. Nöjd, P. Schurtenberger and F. Scheffold, Sci. Adv., 2017, 3, e1700969.

95 T. Colla, R. Blaak and C. N. Likos, Soft Matter, 2018, 14, 5106-5120.

96 J. Riest, P. Mohanty, P. Schurtenberger and C. N. Likos, Z. Phys. Chem., 2012, 226, 711-735.

97 T. Colla and C. N. Likos, Mol. Phys., 2015, 113, 2496-2510.

98 Z. Hu and G. Huang, Angew. Chem., Int. Ed., 2003, 42, 4799-4802.

99 G. Huang and Z. Hu, Macromolecules, 2007, 40, 3749-3756.

100 R. K. Shah, J.-W. Kim and D. A. Weitz, Langmuir, 2009, 26, 1561-1565.

101 C. Urban and P. Schurtenberger, J. Colloid Interface Sci., 1998, 207, 150-158.

102 I. D. Block and F. Scheffold, Rev. Sci. Instrum., 2010, 81, 123107.

103 L. K. Månsson, J. N. Immink, A. M. Mihut, P. Schurtenberger and J. J. Crassous, Faraday Discuss., 2015, 181, 49-69.

104 D. Duracher, A. Elassari and C. Pichot, J. Polym. Sci., Part A: Polym. Chem., 1999, 37, 1823-1837.

105 D. Duracher, A. Elassari and C. Pichot, Colloid Polym. Sci., 1999, 277, 905-913.

106 A. Pich and W. Richtering, in Chemical Design of Responsive Microgels, ed. A. Pich and W. Richtering, Springer, Berlin, Heidelberg, 2010, ch. 1, pp. 1-37.

107 A. Guillermo, J. Cohen Addad, J. Bazile, D. Duracher, A. Elassari and C. Pichot, J. Polym. Sci., Part B: Polym. Phys., 2000, 38, 889-898. 
108 Z. Meng, M. H. Smith and L. A. Lyon, Colloid Polym. Sci., 2009, 287, 277-285.

109 K. Kratz, T. Hellweg and W. Eimer, Colloids Surf., A, 2000, 170, 137-149.

110 M. J. Bergman, J. S. Pedersen, P. Schurtenberger and N. Boon, unpublished work.

111 L. A. Lyon, J. D. Debord, S. B. Debord, C. D. Jones, J. G. McGrath and M. J. Serpe, J. Phys. Chem. B, 2004, 108, 19099-19108.

112 C. S. Wagner, Y. Lu and A. Wittemann, Langmuir, 2008, 24, 12126-12128.

113 C. S. Wagner, B. Fischer, M. May and A. Wittemann, Colloid Polym. Sci., 2010, 288, 487-498.

114 B. Kronberg, K. Holmberg and B. Lindman, in Surface Chemistry of Surfactants and Polymers, ed. B. Kronberg, K. Holmberg and B. Lindman, Wiley, Chichester, 1st edn, 2014, ch. 11, pp. 211-229.

115 H. G. Khorana, Chem. Rev., 1953, 53, 145-166.

116 L. R. Huang, E. C. Cox, R. H. Austin and J. C. Sturm, Science, 2004, 304, 987-990.

117 J. McGrath, M. Jimenez and H. Bridle, Lab Chip, 2014, 14, 4139-4158.

118 S. H. Holm, J. P. Beech, M. P. Barrett and J. O. Tegenfeldt, Anal. Methods, 2016, 8, 3291-3300.

119 S. Ranjan, K. K. Zeming, R. Jureen, D. Fisher and Y. Zhang, Lab Chip, 2014, 14, 4250-4262.

120 K. K. Zeming, T. Salafi, C.-H. Chen and Y. Zhang, Sci. Rep., 2016, 6, 22934.

121 J. P. Beech, S. H. Holm, K. Adolfsson and J. O. Tegenfeldt, Lab Chip, 2012, 12, 1048-1051.

122 J. P. Beech, P. Jönsson and J. O. Tegenfeldt, Lab Chip, 2009, 9, 2698-2706.

123 K. K. Zeming, N. V. Thakor, Y. Zhang and C.-H. Chen, Lab Chip, 2016, 16, 75-85.

124 B. H. Wunsch, J. T. Smith, S. M. Gifford, C. Wang, M. Brink, R. L. Bruce, R. H. Austin, G. Stolovitzky and Y. Astier, Nat. Nanotechnol., 2016, 11, 936-940.
125 S. M. Santana, M. A. Antonyak, R. A. Cerione and B. J. Kirby, Biomed. Microdevices, 2014, 16, 869-877.

126 D. W. Inglis, K. J. Morton, J. A. Davis, T. J. Zieziulewicz, D. A. Lawrence, R. H. Austin and J. C. Sturm, Lab Chip, 2008, 8, 925-931.

127 J. A. Davis, D. W. Inglis, K. J. Morton, D. A. Lawrence, L. R. Huang, S. Y. Chou, J. C. Sturm and R. H. Austin, Proc. Natl. Acad. Sci. U. S. A., 2006, 103, 14779-14784.

128 K. Loutherback, J. D’Silva, L. Liu, A. Wu, R. H. Austin and J. C. Sturm, AIP Adv., 2012, 2, 042107.

129 S. H. Holm, J. P. Beech, M. P. Barrett and J. O. Tegenfeldt, Lab Chip, 2011, 11, 1326-1332.

130 T. S. Tran, B. D. Ho, J. P. Beech and J. O. Tegenfeldt, $L a b$ Chip, 2017, 17, 3592-3600.

131 H. N. Joensson, M. Uhlén and H. A. Svahn, Lab Chip, 2011, 11, 1305-1310.

132 J. P. Beech, B. D. Ho, G. Garriss, V. Oliveira, B. HenriquesNormark and J. O. Tegenfeldt, Anal. Chim. Acta, 2018, 1000, 223-231.

133 J. V. Green, M. Radisic and S. K. Murthy, Anal. Chem., 2009, 81, 9178-9182.

134 J. A. Davis, PhD thesis, Princeton University, 2008.

135 R. Contreras-Cáceres, A. Sánchez-Iglesias, M. Karg, I. Pastoriza-Santos, J. Pérez-Juste, J. Pacifico, T. Hellweg, A. Fernández-Barbero and L. M. Liz-Marzán, Adv. Mater., 2008, 20, 1666-1670.

136 R. Contreras-Cáceres, S. Abalde-Cela, P. Guardia-Girós, A. Fernández-Barbero, J. Pérez-Juste, R. A. Alvarez-Puebla and L. M. Liz-Marzán, Langmuir, 2011, 27, 4520-4525.

137 M. Karg and T. Hellweg, Curr. Opin. Colloid Interface Sci., 2009, 14, 438-450.

138 C. Dagallier, H. Dietsch, P. Schurtenberger and F. Scheffold, Soft Matter, 2010, 6, 2174-2177.

139 J. J. Crassous, A. M. Mihut, H. Dietsch, O. Pravaz, L. Ackermann-Hirschi, A. M. Hirt and P. Schurtenberger, Nanoscale, 2014, 6, 8726-8735. 